Revista Brasileira de Meteorologia, v.25, n.3, 365 - 374, 2010

\title{
OBTENÇÃO DOS FLUXOS DE ENERGIA À SUPERFÍCIE UTILIZANDO O ALGORITMO S-SEBI
}

\author{
CARLOS ANTONIO COSTA DOS SANTOS E BERNARDO BARBOSA DA SILVA
}

\author{
Unidade Acadêmica de Ciências Atmosféricas, Universidade Federal de Campina Grande, \\ Campina Grande, UFCG, PB, Brasil.
}

carlostorm@gmail.com carlos@dca.ufcg.edu.br, bernardo@dca.ufcg.edu.br

Recebido Maio 2009 - Aceito Fevereiro 2010

\begin{abstract}
RESUMO
O principal objetivo desse estudo foi contribuir para a compreensão das estimativas e análises espaciais dos fluxos de energia à superfície, utilizando o algoritmo S-SEBI e imagens Landsat 5 - TM, assim como, validar os resultados com medidas obtidas na torre micrometeorológica. A área de estudo foi a fazenda Frutacor, com área plantada com cultivos de bananeiras e áreas adjacentes com solo exposto e vegetação nativa. A referida área fica localizada no município de Quixeré, na microrregião do Baixo Jaguaribe, Estado do Ceará. O algoritmo S-SEBI apresentou-se como um bom estimador dos fluxos de energia à superfície, apresentando concordância com estudos desenvolvidos em diferentes partes do mundo. A comparação dos resultados estimados pelo S-SEBI com os medidos na torre micrometeorológica mostrou que os parâmetros, estimados pelo referido algoritmo, estão em boa concordância com os medidos, evidenciando assim, que o S-SEBI é uma ferramenta promissora na obtenção da distribuição espacial dos fluxos de energia à superfície em regiões semi-áridas.
\end{abstract}

Palavras-Chave: Saldo de radiação, banana, vegetação nativa, semi-árido

\begin{abstract}
SURFACE ENERGY FLUXES ESTIMATIVE USING THE S-SEBI ALGORITHM The main objective of this study was to contribute to the understanding of the estimates and spatial analyses of the surface energy fluxes using the S-SEBI algorithm and TM Landsat 5 images, and to validate the measurements obtained at the micrometeorological tower. The study area was the Frutacor farm, with cultivated area of banana crops, adjacent to bare soil and native vegetation areas, located at the irrigation district of Quixeré, in the Low Jaguaribe basin, Ceará State. The S-SEBI algorithm seems to be a good estimator of the surface energy fluxes in agreement to different studies developed throughout the world. The comparison between the S-SEBI and the micrometeorological results showed good agreement, evidencing that the S-SEBI algorithm is a promising tool in obtaining the spatial distribution of the surface energy fluxes over semi-arid regions.
\end{abstract}

Keywords: Net radiation, banana crop, native vegetation, semiarid

\section{INTRODUÇÃO}

O estudo das trocas de energia e água entre a superfície da terra e a atmosfera é de extrema importância para entender as mudanças no meio ambiente e no clima. Pode ajudar a entender também a melhorar a precisão da modelagem climática (Fan et al., 2007), como também, no manejo adequado das atividades que envolvem o uso de água, por exemplo, a agricultura irrigada (Teixeira et al., 2009). Segundo Roerink et al. (2000), os processos de trocas que ocorrem na superfície da terra são de crucial importância para a redistribuição da umidade e calor no solo e atmosfera. Para melhor entender esses processos de mudanças, a utilização das informações de sensoriamento remoto por satélite é indispensável para extrapolar medidas pontuais em uma escala regional. Os dados do satélite Landsat-TM podem ser comparados diretamente com medidas de campo, devido a sua alta resolução espacial.

Recentemente, têm sido propostos métodos que obtêm o Balanço de Energia (BE) à superfície, a partir de dados de sensoriamento remoto orbital. Este é um caminho promissor, 
pois as estimativas do saldo de radiação (Rn) e dos fluxos de calor no solo (G), sensível (H) e latente (LE) geradas a partir desses dados, oferecem a vantagem de apresentá-los distribuídos de forma espacial, ou seja, com resultados diferenciados e inerentes a cada tipo de cobertura vegetativa presente na cena. Outra grande vantagem é que não são necessárias campanhas experimentais de campo, nem conhecimento prévio da cultura nem do sistema de cultivo. A maioria desses métodos requer apenas dados de estações meteorológicas, como velocidade do vento e temperatura do ar do instante da passagem do satélite, localizadas no interior da cena estudada (Sobrino et al., 2007).

De acordo com Gómez et al. (2005) o conhecimento detalhado dos fluxos à superfície, especialmente as componentes de calor sensível e latente, é de interesse primário para aplicações ambientais: monitoramento do clima, avaliação dos esquemas de parametrizações para modelos climáticos e de tempo, recursos hídricos (Boronina e Ramillien, 2008) e avaliação da irrigação para aperfeiçoar práticas agrícolas (Tasumi e Allen, 2007; Zwart e Bastiaanssen, 2007, Teixeira et al., 2009).

Dentre os métodos que têm sido desenvolvidos para derivar os fluxos de energia na superfície, utilizando dados gerados por sensoriamento remoto orbital, destacam-se os algoritmos SEBAL (Surface Energy Balance Algorithm for Land, Bastiaanssen et al., 1998 e Bastiaanssen, 2000), SEBS (Surface Energy Balance System, Su, 2002), TSEB (Two-Source Energy Balance, Norman et al., 1995). Basicamente, a diferença entre os métodos está no cálculo do fluxo de calor sensível (H). Os modelos SEBS e o SEBAL, em particular, propiciam estes cálculos a partir de métodos bastantes complexos baseados na teoria da similaridade de Monin-Obukhov e, necessitam de dados meteorológicos adicionais (Bastiaanssen et al., 1998).

Um novo método, relativamente simples, para derivar os fluxos do BE através de medidas por sensoriamento remoto, chama-se o Simplified Surface Energy Balance Index (S-SEBI) que foi desenvolvido, testado e validado com medidas pontuais dos fluxos (Roerink et al., 2000). Basicamente se determina a reflectância através da temperatura máxima para condições secas, e a reflectância também dependente da temperatura mínima para condições úmidas. Em seguida, os fluxos de calor sensível e latente são particionados. Esta metodologia apresentada é bastante simplificada, e aparentemente oferece maior operacionalidade em relação às demais metodologias. Os cálculos do H e LE são feitos a partir da fração evaporativa, que por sua vez é obtida com base na reflectância e na temperatura da superfície, pixel a pixel (Roerink et al., 2000). O algoritmo S-SEBI foi validado na Itália (Roerink et al., 2000), na Espanha (Gómez et al., 2005; Sobrino et al., 2005), na Holanda (Weligepolage, 2005) e na Península Ibérica (Sobrino et al., 2007). O mesmo foi aplicado para uma região semi-árida no
Nordeste do Brasil (Santos et al., 2007; Santos e Silva, 2008), no entanto, os fluxos de energia obtidos através desse método ainda não foram validados no Brasil.

Assim, com o intuito de contribuir para a compreensão das estimativas e análises espaciais dos fluxos de energia à superfície, este estudo tem como objetivo utilizar a metodologia proposta por Roerink et al. (2000) para estimar a distribuição espacial de Rn, G, H e LE, em áreas irrigadas, de vegetação nativa e solo exposto, a partir de imagens de satélite Landsat 5 - TM e validar os resultados com medidas obtidas na torre micrometeorológica.

\section{MATERIAL E MÉTODOS 2.1 Área de Estudo}

A área de estudo foi a fazenda Frutacor, com área aproximada de 250 ha de cultivo de bananeiras (Musa sp.) e áreas adjacentes com solo exposto e vegetação nativa (Caatinga), situada a $5^{\circ} 08^{\prime} 44^{\prime \prime} \mathrm{S}$ e $38^{\circ} 05^{\prime} 53^{\prime \prime} \mathrm{W}$, apresentando altitude de aproximadamente $147 \mathrm{~m}$. A referida área fica localizada no município de Quixeré, na microrregião do Baixo Jaguaribe, Estado do Ceará, conforme ilustrado na Figura 1a. A região apresenta clima quente e semi-árido, do tipo BSw'h', de acordo com a classificação de Köeppen. Sua temperatura média anual é de $28,5^{\circ} \mathrm{C}$, sendo as temperaturas máximas e mínimas anuais de $36^{\circ} \mathrm{C}$ e $22^{\circ} \mathrm{C}$, respectivamente. A precipitação média anual da região é de $772 \mathrm{~mm}$, cuja distribuição média mensal de 25 anos (1981 - 2006) registrado no posto pluviométrico de Quixeré-CE está ilustrada na Figura $1 b$.

Uma estação meteorológica automática (106 Weather Station, Campbell Scientific Inc., Logan, UT, USA) foi instalada na área de estudo para obter a evapotranspiração de referência $\left(\mathrm{ET}_{0}\right)$ pelo método combinado da FAO - Penman-Monteith (Allen et al., 1998). O saldo de radiação (Rn) foi computado usando um saldo radiômetro (CNR1, Kipp \& Zonnen, Delf, The Netherlands), instalado a 7,0 $\mathrm{m}$ acima da superfície e 2,0 m acima do dossel da cultura (Tabela 1). A temperatura e umidade relativa do ar foram medidas usando um sensor da Vaisala HMP45C. O fluxo de calor no solo $(\mathrm{G})$ foi medido com dois fluxímetros (HFP01SC-L, Campbell Scientific, Inc., Logan, UT) instalados a uma profundidade de $2,0 \mathrm{~cm}$ (Tabela 1), uma entre plantas e a outra entre fileiras. Os valores de $\mathrm{G}$ foram obtidos através da média entre os dois fluxímetros. Um sistema de correlações turbulentas foi utilizado na obtenção das medidas dos fluxos turbulentos, sendo composto de um analisador de gás infravermelho (IRGA) (LI-7500, Licor, Inc., Lincoln, NE) acoplado a um anemômetro sônico tridimensional (CSAT-3, Campbell Scientific, Inc., Logan, UT), usado para medir as flutuações dos componentes da velocidade do vento. Os sinais digitais desses instrumentos foram obtidos numa frequência 
de $10 \mathrm{~Hz}$ e, as médias obtidas em intervalos de 30 minutos usando um datalogger CR23X da Campbell Scientific, onde os dados foram armazenados para o posterior processamento. $\mathrm{Na}$ análise dos dados foram aplicadas somente as correções de Webb (Webb et al., 1980) e as correções do vento transversal (Tanaka et al., 2003).

\subsection{Metodologia}

Neste estudo, utilizou-se imagem do sensor Thematic Mapper (TM) a bordo do satélite Landsat-5, referentes à órbita/ ponto 216/64, do dia 24/10/2005. As variáveis da superfície da terra e da vegetação, tais como: albedo da superfície $\left(\mathrm{r}_{0}\right)$, temperatura da superfície $\left(\mathrm{T}_{\mathrm{s}}\right)$, emissividade termal da superfície (ع), Índice de Vegetação por Diferença Normalizada (NDVI) e Índice de Área Foliar (LAI), assim como, os fluxos de energia à superfície foram derivadas a partir de dados obtidos do satélite. A seguir estão apresentadas as informações sobre o método aplicado neste estudo:

A primeira etapa computacional do S-SEBI foi a obtenção da calibração radiométrica ou radiância espectral, $\left(L_{\lambda}\right)$, calculada pela seguinte expressão:

$$
L_{\lambda}=\left(\frac{L_{M A X}-L_{M I N}}{255}\right) \times D N-L_{M I N}
$$

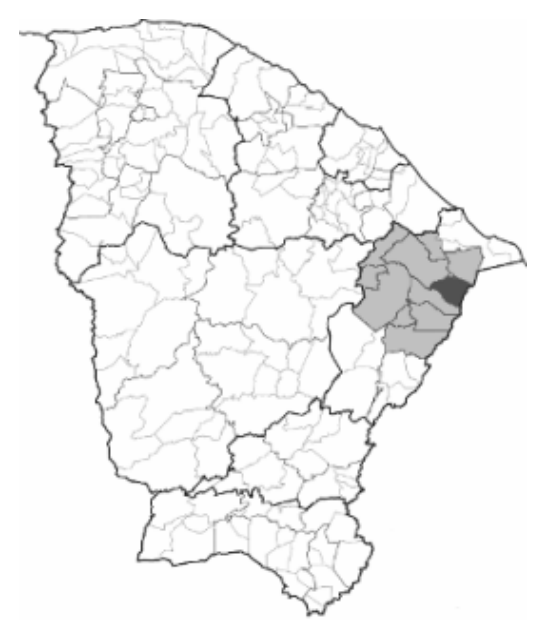

onde DN é o número digital de cada pixel da imagem, $\mathrm{L}_{\mathrm{MAX}} \mathrm{e}$ $\mathrm{L}_{\mathrm{MIN}}$ são as constantes de calibração espectral do sensor, cujos valores para o Landsat 5-TM, segundo Chander e Markham (2003), são apresentados na Tabela 2.

Em seguida, calculou-se a reflectância monocromática $\left(\rho_{\lambda}\right)$, obtida pela equação (Allen et al., 2002):

$$
\rho_{\lambda}=\frac{\pi L_{\lambda}}{E S U N_{\lambda} \cos \theta d_{r}}
$$

onde $\mathrm{L}_{\lambda}$ é a radiância espectral; $E S U N_{\lambda}$ é a constante solar monocromática associada a cada banda do sensor, cujos valores válidos para dados radiométricos gerados pelo Landsat 5 após 5 de maio de 2003, de acordo com Chander e Markham (2003), estão apresentados na Tabela 3; $\theta$ é o ângulo zenital do Sol; e $d_{r}$ é o inverso do quadrado da distância Terra-Sol, que é obtida pela Equação 3.

$$
d_{r}=1+0,033 \cos \left(D O A \frac{2 \pi}{365}\right)
$$

onde DOA é o dia de ordem do ano em que são obtidos os dados radiométricos (imagens de satélite), cujos valores correspondem ao dia da geração das imagens.

O cálculo do albedo no topo da atmosfera foi feito através de uma combinação linear das reflectâncias espectrais

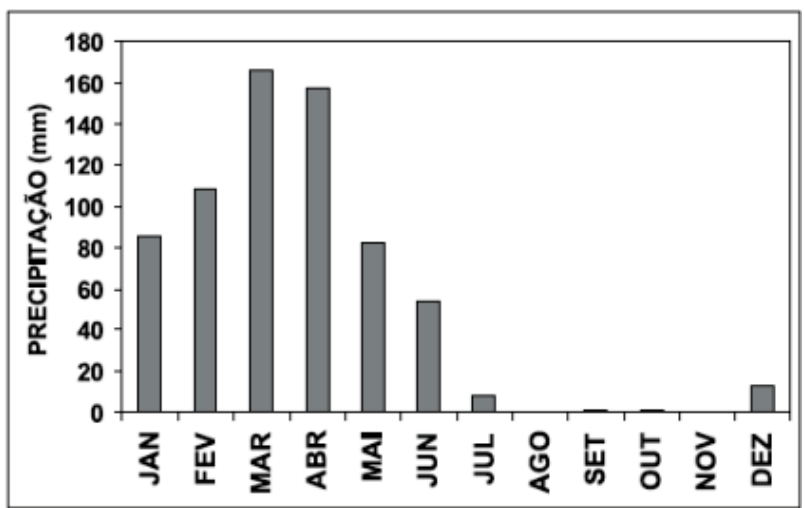

Figura 1 - a) Mapa do Estado do Ceará onde aparece destacada a microrregião de Baixo Jaguaribe e o município de Quixeré; b) Distribuição das médias mensais da precipitação de Quixeré-CE (1981 - 2006).

Tabela 1 - Instrumentos instalados na torre micrometeorológica contendo o sistema de correlações turbulentas

\begin{tabular}{cllc}
\hline Sistema & \multicolumn{1}{c}{ Variáveis } & \multicolumn{1}{c}{ Instrumentos } & Altura (m) \\
\hline Correlações turbulentas & Velocidade do vento & CSAT3 3D sonic anemometer & 7,0 \\
& Temperatura e umidade & HMP45C probe & 7,0 \\
& relativa do ar & Licor 7500 & 7,0 \\
& $\mathrm{CO}_{2}$ e $\mathrm{H}_{2} \mathrm{O}$ & HFP01SC-L & 0,02 \\
\hline
\end{tabular}


$\left(\rho_{\lambda}\right)$, calculados anteriormente, e o coeficiente de regressão $\left(\varpi_{\lambda}\right)$, para cada banda de acordo com a equação seguinte:

$$
\begin{aligned}
& \alpha_{\text {toa }}=\left(\varpi_{1} \times \rho_{1}\right)+\left(\varpi_{2} \times \rho_{2}\right)+\left(\varpi_{3} \times \rho_{3}\right) \\
& +\left(\varpi_{4} \times \rho_{4}\right)+\left(\varpi_{5} \times \rho_{5}\right)+\left(\varpi_{7} \times \rho_{7}\right)
\end{aligned}
$$

Os coeficientes de regressão linear da equação anterior $\left(\varpi_{1}, \varpi_{2}, \ldots \bar{\varpi}_{7}\right)$, para o Landsat 5-TM, são apresentados na Tabela 4:

Tabela 2 - Instrumentos instalados na torre micrometeorológica contendo o sistema de correlações turbulentas

\begin{tabular}{ccc}
\hline Banda & $\mathbf{L}_{\mathbf{M I N}}\left(\mathbf{W} \mathbf{~ m}^{-\mathbf{2}} \mathbf{s t e r}^{\mathbf{1}} \boldsymbol{\mu m}^{-\mathbf{1}}\right)$ & $\mathbf{L}_{\mathbf{M A X}}\left(\mathbf{W} \mathbf{~ m}^{-\mathbf{2}} \mathbf{s t e r}^{\mathbf{1}} \boldsymbol{\mu} \mathbf{m}^{-\mathbf{1}}\right)$ \\
\hline 1 & $-1,52$ & 193,0 \\
2 & $-2,84$ & 365,0 \\
3 & $-1,17$ & 264,0 \\
4 & $-1,51$ & 221,0 \\
5 & $-0,37$ & 30,2 \\
6 & 1,24 & 15,3 \\
7 & $-0,15$ & 16,5 \\
\hline
\end{tabular}

O albedo da superfície foi computado através da equação seguinte:

$$
r_{0}=\frac{\alpha_{\text {toa }}-\alpha_{\text {path_radiance }}}{\tau_{s v}^{2}}
$$

onde $\alpha_{\text {toa }}$ é o albedo planetário, $\alpha_{\text {path_radiance é a porção da }}$ radiação solar refletida pela atmosfera (Bastiaanssen, 2000) e $\tau_{\mathrm{sw}}$ é a transmissividade atmosférica, que é definida como a fração da radiação solar incidente transmitida pela atmosfera e representa o seu efeito de absorção e reflecção (Allen et al., 2002). A transmissividade atmosférica foi computada em função da altitude local (z), conforme a equação:

$$
\tau_{s w}=0,75+2 \times 10^{-5} \times z
$$

O NDVI, que é um indicativo das condições, densidade e porte da vegetação, foi obtido através da razão entre a diferença das reflectâncias do infravermelho próximo $\left(\rho_{4}\right)$ e do vermelho $\left(\rho_{3}\right)$ e a soma das mesmas reflectâncias conforme equação (Allen et al., 2002):

$$
N D V I=\left(\rho_{4}-\rho_{3}\right) /\left(\rho_{4}+\rho_{3}\right)
$$

O SAVI é um índice de vegetação que visa amenizar os efeitos de "background" do solo, sendo obtido através da equação a seguir (Huete, 1988):

$$
S A V I=(1+L)\left(\rho_{4}-\rho_{3}\right) /\left(L+\rho_{4}+\rho_{3}\right)
$$

onde Lé um fator de ajuste ao solo $(\mathrm{L}=0,5)$ (Allen et al., 2002).

$\mathrm{O}$ cômputo do LAI, que representa a razão entre a área total de todas as folhas contidas em dado pixel, pela área do pixel, é feito por equação empírica obtida por Allen et al. (2002):

$$
L A I=-\frac{\ln \left(\frac{0,69-S A V I}{0,59}\right)}{0,91}
$$

O cálculo da temperatura da superfície foi feito através da equação de Planck invertida, proposta para um corpo negro, para o qual a emissividade é igual a 1. Como cada pixel não constitui um corpo negro, há necessidade de se estabelecer a sua emissividade no domínio espectral da banda termal $\left(\varepsilon_{\mathrm{NB}}\right)$, para fins do cômputo da $T_{s}$, bem como no cômputo da radiação de onda longa emitida pela superfície, que nesse caso é considerada a emissividade da banda larga $\left(\varepsilon_{0}\right)(4-100 \mu \mathrm{m})$.

O cômputo de ambas as emissividades foi feito através das equações seguintes em função do LAI:

$$
\begin{aligned}
& \varepsilon_{N B}=0,97+0,00331 L A I \\
& \varepsilon_{0}=0,95+0,01 L A I
\end{aligned}
$$

Foram considerados $\varepsilon_{\mathrm{NB}}=\varepsilon_{0}=0,98$ quando LAI 3 , e para NDVI $<0$, no caso de corpos de água, considera-se $\varepsilon_{\mathrm{NB}}=$ 0,99 e $\varepsilon_{0}=0,985$, de acordo com proposta de Allen et al. (2002).

Tabela 3 - Valores do $E S U N_{1}\left(\mathrm{~W} \mathrm{~m}^{-2} \mu \mathrm{m}^{-1}\right)$ válidos para o Landsat 5 - TM

\begin{tabular}{cccccccc}
\hline & Banda 1 & Banda 2 & Banda 3 & Banda 4 & Banda 5 & Banda 6 & Banda 7 \\
\hline Landsat 5 & 1957 & 1826 & 1554 & 1036 & 215,0 & - & 80,67 \\
\hline
\end{tabular}

Tabela 4 - Instrumentos instalados na torre micrometeorológica contendo o sistema de correlações turbulentas

\begin{tabular}{cccccccc}
\hline & $\boldsymbol{\varpi}_{1}$ & $\boldsymbol{\varpi}_{2}$ & $\boldsymbol{\varpi}_{3}$ & $\boldsymbol{\varpi}_{4}$ & $\varpi_{5}$ & $\varpi_{6}$ & $\varpi_{7}$ \\
\hline Landsat 5-TM & 0,293 & 0,274 & 0,233 & 0,157 & 0,033 & - & 0,011 \\
\hline
\end{tabular}


A obtenção de $T_{s}$, como mencionado anteriormente, foi feita através da equação de Planck invertida, em função da radiância espectral da banda termal $\mathrm{L}_{\lambda, 6} \mathrm{e}$ da emissividade obtida pela equação (10a):

$$
T_{s}=\frac{K_{2}}{\ln \left(\frac{\varepsilon_{N B} K_{1}}{L_{\lambda, 6}}+1\right)}
$$

onde $K_{1}=607,8$ e $K_{2}=1261 \mathrm{Wm}^{-2} \mathrm{sr}^{-1} \mu \mathrm{m}^{-1}$.

O fluxo de radiação termal emitida pela superfície foi obtido pela equação de Stefan-Boltzmann, em função da temperatura e da emissividade da superfície.

$$
R_{L \uparrow}=\varepsilon_{0} \sigma T_{s}^{4}
$$

onde $\sigma$ é constante de Boltzmann $\left(5,67 \times 10^{-8} \mathrm{~W} \mathrm{~m}^{-2} \mathrm{~K}^{-4}\right)$ e os outros termos já foram definidos anteriormente.

A radiação de onda longa emitida pela atmosfera na direção da superfície $\left(\mathrm{W} \mathrm{m}^{-2}\right)$, também foi calculada pela equação de Stefan-Boltmann, em função da emissividade do ar que foi obtida pela equação (14) e da temperatura do $\operatorname{ar}\left(\mathrm{T}_{\mathrm{a}}\right)$, obtida junto a uma estação meteorológica, na área de estudo:

$$
R_{L \downarrow}=\varepsilon_{a} \sigma T_{a}^{4}
$$

A radiação de onda curta incidente $R_{s \downarrow}$ foi calculado pela equação:

$$
\varepsilon_{a}=0,85\left(-\ln \tau_{s w}\right)^{0,09}
$$

onde $\mathrm{G}_{\mathrm{sc}}$ é a constante solar $\left(1367 \mathrm{~W} \mathrm{~m}^{-2}\right)$ e os demais termos já foram previamente definidos.

O componente Rn utilizado no algoritmo S-SEBI, foi obtido pela equação a seguir (Allen et al., 2002; Allen et al., 2005; Tasumi et al., 2005).

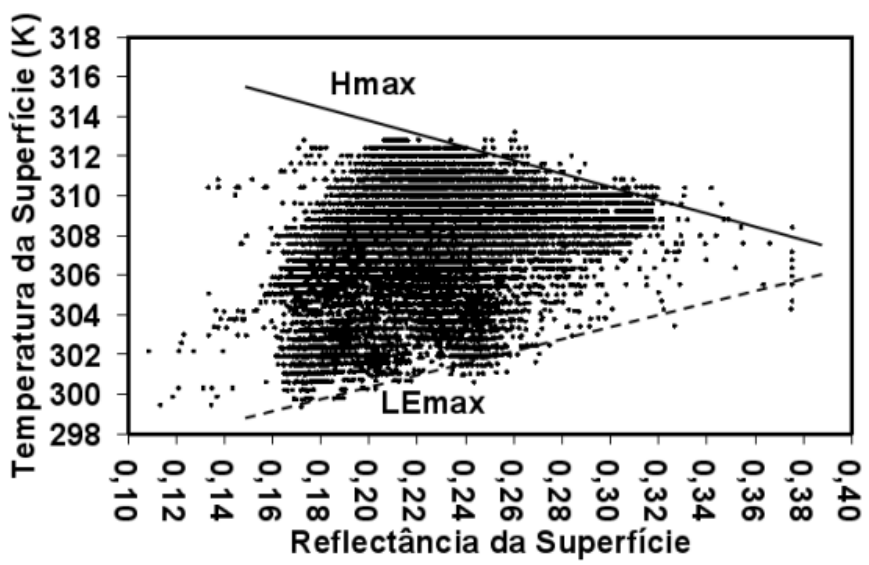

Figura 2 - Representação esquemática da relação entre a temperatura da superfície e a reflectância da superfície junto com princípios básicos do S-SEBI.

$$
R_{s \downarrow}=G_{s c} \cos \theta d_{r} \tau_{s w}
$$

A densidade de fluxo de calor no solo, $G$, foi obtida mediante metodologia proposta por Bastiaanssen (2000).

$$
R n=(1-\alpha) R_{S \downarrow}+R_{L \downarrow}-R_{L \uparrow}-\left(1-\varepsilon_{0}\right) R_{L \downarrow}
$$

onde $\alpha$ é o albedo, $\mathrm{T}_{\mathrm{s}}$ é a temperatura da superfície e NDVI é o índice de vegetação da diferença normalizada.

A principal diferença entre S-SEBI e os demais algoritmos, citados anteriormente, está na obtenção dos fluxos de $\mathrm{H}$ e LE, uma vez que os demais algoritmos necessitam de informações de superfície e se utilizam de metodologias complexas para a obtenção do fluxo de calor sensível. No S-SEBI os fluxos $\mathrm{H}$ e $\mathrm{LE}\left(\mathrm{W} \mathrm{m}^{-2}\right)$ foram obtidos a partir da fração evaporativa $(\Lambda)$.

No entanto, o cálculo da fração evaporativa para cada pixel com reflectância $\alpha$ e temperatura $T_{s}$ foi efetuado conforme metodologia de Roerink et al. (2000). Assim, $\Lambda$ para um pixel particular foi calculada como a razão dada por:

$$
\Lambda=\frac{T_{H}-T_{s}}{T_{H}-T_{L E}}
$$

A Figura 2 mostra as características espaciais da reflectância e da temperatura da superfície. $\mathrm{Na}$ distribuição espacial é possível reconhecer o limite inferior, onde o fluxo de calor latente máximo $\mathrm{LE}_{\max }(\alpha)$ é assumido e, o limite superior, onde o fluxo de calor sensível máximo $\mathrm{H}_{\max }(\alpha)$ é assumido (Sobrino et al., 2007). As equações lineares que representam os limites máximos e mínimos podem ser escritas como:

$$
\begin{aligned}
& T_{H}=a_{H}+b_{H} \alpha \\
& T_{L E}=a_{L E}+b_{L E} \alpha
\end{aligned}
$$

As variáveis de regressão $a$ e $b$ são locais e temporais, ou seja, variam no tempo e no espaço.

Substituindo a relação observada por $\mathrm{T}_{\mathrm{H}} \mathrm{e} \mathrm{T}_{\mathrm{LE}}$, a fração evaporativa pode ser escrita como:

$$
\Lambda=\frac{a_{H}+b_{H} \alpha-T_{s}}{a_{H}-a_{L E}+\left(b_{H}-b_{L E}\right) \alpha}
$$

Uma vez determinada $\Lambda$, os fluxos de calor sensível e latente foram calculados conforme descrito abaixo, seguindo a metodologia de Roerink et al. (2000):

$$
\begin{aligned}
& H=(1-\Lambda)\left(R_{n}-G\right) \\
& L E=\Lambda\left(R_{n}-G\right)
\end{aligned}
$$




\section{RESULTADOS E DISCUSSÃO}

A Tabela 5 apresenta a variação regional do albedo da superfície, NDVI e fluxos de energia a superfície (Rn, H, LE e G), juntamente com os valores médios e desvio-padrão, para os diferentes tipos de cobertura do solo, do albedo da superfície e NDVI utilizando imagem Landsat 5-TM e o algoritmo S-SEBI, para a cena escolhida (dia 24/10/2005). O albedo superficial variou entre 0,1 e 0,37 , o NDVI entre 0,0 e 0,82 , podendo ser entendidos como representativos para áreas de solo exposto e de total cobertura do solo por vegetação, respectivamente. A temperatura da superfície variou entre 26,1 e $40,0^{\circ} \mathrm{C}$ evidenciando os diferentes tipos de cobertura do solo. Os fluxos de energia à superfície variaram de 429,3 a $734,7 \mathrm{~W} \mathrm{~m}^{-2}, 57,6$ a $220,4 \mathrm{~W} \mathrm{~m}^{-2}, 0,4$ a $434,7 \mathrm{~W} \mathrm{~m}^{-2}$ e entre 0,2 e $604,2 \mathrm{~W} \mathrm{~m}^{-2}$ para os Rn, G, He LE, respectivamente. Os resultados mostram a alta variabilidade na cobertura do solo, indo do solo exposto a vegetação densa para as áreas irrigadas plantadas com a cultura da banana.

Devido à alta variabilidade da cobertura do solo, observa-se ainda que o uso do solo foi dividido em três classes que são: solo exposto, vegetação nativa (Caatinga) e pomar de bananas. O solo exposto apresentou um albedo médio de 0,21 e um NDVI médio de 0,11, sendo que ambos apresentaram baixos valores de desvio-padrão. A temperatura média da superfície foi de $36,7^{\circ} \mathrm{C}$ com um desvio-padrão de $0,3^{\circ} \mathrm{C}$. Os fluxos de energia médios foram de $576,5 \mathrm{~W} \mathrm{~m}^{-2}$, $116,5 \mathrm{~W} \mathrm{~m}^{-2}, 366,3 \mathrm{~W} \mathrm{~m}^{-2}$ e 93,6 $\mathrm{W} \mathrm{m}^{-2}$, correspondendo ao $\mathrm{Rn}, \mathrm{G}, \mathrm{H}$ e LE, respectivamente, onde todos mostraram baixos valores de desvio-padrão evidenciando a homogeneidade da área de solo exposto estudada. A área de vegetação nativa apresentou valores médios para o albedo de 0,18 e de NDVI de 0,29 , ambos apresentando baixa variabilidade nos valores. A temperatura média da superfície foi de $34,0^{\circ} \mathrm{C}$ com um desvio-padrão de $0,1^{\circ} \mathrm{C}$. A vegetação nativa da região é esparsa e de baixo porte, podendo ser identificado na análise da temperatura da superfície, visto que a temperatura média para o solo exposto foi próximo da temperatura média da vegetação nativa, evidenciando que existe pouca cobertura do solo pela vegetação. Os fluxos de energia médios foram de $628,8 \mathrm{~W} \mathrm{~m}^{-2}, 109,1 \mathrm{~W} \mathrm{~m}^{-2}, 267,8 \mathrm{~W} \mathrm{~m}^{-2}$ e $251,9 \mathrm{~W} \mathrm{~m}^{-2}$, correspondendo ao Rn, G, H e LE, respectivamente, todos apresentam baixos valores de desvio-padrão. A área irrigada do pomar de bananeiras apresentou um albedo médio de 0,17 e um NDVI médio de 0,66 , evidenciando a alta densidade e porte da cultura estudada. A temperatura média da superfície foi de $28,9^{\circ} \mathrm{C}$, com um desvio-padrão de $0,3^{\circ} \mathrm{C}$. Os fluxos de energia médios foram de $666,7 \mathrm{~W} \mathrm{~m}^{-2}, 78,7 \mathrm{~W} \mathrm{~m}^{-2}, 110,6$

Tabela 5 - Variação regional e valores médios com o desvio-padrão para os diferentes tipos de cobertura do solo, para o albedo, NDVI e fluxos de energia a superfície, utilizando imagem Landsat 5-TM

\begin{tabular}{|c|c|c|c|c|c|c|c|}
\hline & $\mathbf{r}_{0}$ & NDVI & $\begin{array}{c}\mathrm{T}_{5} \\
\left({ }^{\circ} \mathrm{C}\right)\end{array}$ & $\begin{array}{c}\mathbf{R n} \\
\left(\mathbf{W ~ m ~ m}^{-2}\right)\end{array}$ & $\begin{array}{c}\mathbf{G} \\
\left(\mathbf{W ~ m}^{-2}\right)\end{array}$ & $\begin{array}{c}\mathbf{H} \\
\left(\mathbf{W ~ m}^{-2}\right)\end{array}$ & $\begin{array}{c}\mathrm{LE} \\
\left(\mathrm{W} \mathrm{m}^{-2}\right)\end{array}$ \\
\hline Variação regional & $0,10-0,37$ & $0,0-0,82$ & $26,1-40,0$ & $429,3-734,7$ & $57,6-220,4$ & $0,4-434,7$ & $0,2-604,2$ \\
\hline Solo exposto & $0,21 \pm 0,01$ & $0,11 \pm 0,01$ & $36,7 \pm 0,3$ & $576,5 \pm 6,35$ & $116,5 \pm 0,5$ & $366,3 \pm 8,6$ & $93,6 \pm 13,5$ \\
\hline Vegetação nativa & $0,18 \pm 0,00$ & $0,29 \pm 0,01$ & $34,0 \pm 0,1$ & $628,8 \pm 1,65$ & $109,1 \pm 0,3$ & $267,8 \pm 3,1$ & $251,9 \pm 3,5$ \\
\hline Pomar de bananas & $0,17 \pm 0,01$ & $0,66 \pm 0,05$ & $28,9 \pm 0,3$ & $666,7 \pm 4,46$ & $78,7 \pm 5,1$ & $110,6 \pm 13,4$ & $477,4 \pm 13,3$ \\
\hline
\end{tabular}

Tabela 6 - Comparação entre os valores estimados pelo S-SEBI e os medidos na torre micrometeorológica, juntamente com o erro percentual, para o pomar de bananeiras

\begin{tabular}{lccc}
\hline & S-SEBI & Medido & $\begin{array}{c}\text { Erro Percentual } \\
(\%)\end{array}$ \\
\hline $\mathbf{r}_{\mathbf{0}}$ & 0,19 & 0,16 & 18,8 \\
$\mathbf{T}_{5}\left({ }^{\circ} \mathbf{C}\right)$ & 29,6 & 29,8 & $-0,7$ \\
$\mathbf{R n}\left(\mathbf{W m}^{-2}\right)$ & 643,1 & 662,0 & $-2,9$ \\
$\mathbf{G}\left(\mathbf{W m}^{-2}\right)$ & 88,6 & 80,2 & 10,5 \\
$\mathbf{H}\left(\mathbf{W m}^{-2}\right)$ & 114,0 & 167,4 & $-31,9$ \\
$\mathbf{L E}\left(\mathbf{W m}^{-2}\right)$ & 440,6 & 414,1 & 6,4 \\
\hline
\end{tabular}


$\mathrm{W} \mathrm{m} \mathrm{m}^{-2}$ e 477,4 $\mathrm{W} \mathrm{m}^{-2}$, correspondendo ao Rn, G, H e LE, respectivamente, onde todos mostraram baixos valores de desvio-padrão evidenciando a homogeneidade da área com cultivos de banana irrigados.

Na Tabela 6 estão apresentadas as comparações entre os valores estimados pelo S-SEBI e os medidos na torre micrometeorológica pela técnica das correlações turbulentas, juntamente com o erro percentual, do albedo, temperatura da superfície e dos valores dos fluxos de energia à superfície para o pomar de bananeiras. O algoritmo S-SEBI apresentou-se como um bom estimador dos parâmetros citados acima, mostrando um erro de $18,8 \%$ para o albedo da superfície e de $0,7 \%$ para a temperatura da superfície. Na obtenção dos fluxos de energia o S-SEBI apresentou um erro de 2,9\%, 10,5\%, $31,9 \%$ e $6,4 \%$, correspondendo aos fluxos de Rn, G, H e LE, respectivamente. Os resultados acima estão em concordância com os obtidos por Roerink et al. (2000), Weligepolage (2005), Fan et al. (2007) e García et al. (2007).

$\mathrm{Na}$ Figura 3 é possível observar o comportamento diurno dos fluxos de energia à superfície para o dia 24/10/2005, com destaque para o momento do mapeamento do satélite sobre a área estudada. $\mathrm{O}$ Rn alcançou valores máximos da ordem de $700 \mathrm{~W} \mathrm{~m}^{-2}$, enquanto $\mathrm{G}, \mathrm{H}$ e LE alcançaram valores máximos da ordem de 100, 350 e $480 \mathrm{~W} \mathrm{~m}^{-2}$, respectivamente. Como o S-SEBI necessita apenas das informações obtidas através da imagem de satélite (reflectância e temperatura da superfície) na obtenção dos fluxos de H e LE, há uma necessidade de se aplicar o método apenas para condições de céu-claro, haja vista que a presença de nuvens acarreta em um aumento do albedo e uma diminuição da temperatura quando comparado aos valores à superfície.

A distribuição espacial do albedo da superfície está apresentada na Figura 4. Pode ser observado que os valores variaram de 0 (áreas claras) aos superiores a 0,30 (áreas escuras). É possível identificar as áreas de cultivos irrigados, assim como, de solo exposto e as áreas irrigadas que utilizam a técnica da plasticultura apresentando altos valores de albedo. Na Figura 5

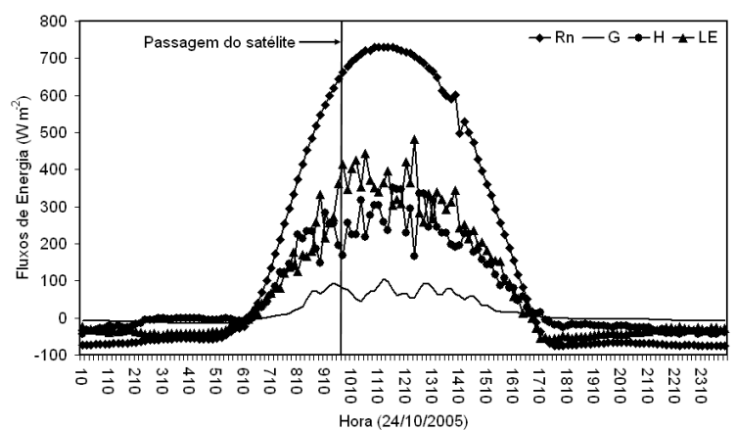

Figura 3 - Distribuição diurna dos fluxos de energia à superfície para o dia 24/10/2005, com destaque para o momento da passagem do satélite na área estudada. está apresentada a distribuição espacial do NDVI, com valores variando de 0 (áreas claras) aos superiores a 0,70 (áreas escuras). Claramente identificam-se as áreas de cultivos irrigados com altos valores de NDVI, assim como, as áreas de vegetação nativa, com valores variando entre 0,20 e 0,40 . Pode ser observada na Figura 6 a distribuição espacial da temperatura da superfície. Os valores variam entre 25 e $40^{\circ} \mathrm{C}$, com predominância de áreas escuras, que representam altos valores de temperatura, evidenciando áreas de solo exposto e de vegetação nativa. É fácil a identificação das áreas com cultivos irrigados, apresentando os menores valores de temperatura da superfície $\left({ }^{\circ} \mathrm{C}\right)$.

As distribuições espaciais dos fluxos de energia à superfície estão apresentadas nas Figuras 7 a 10. A distribuição espacial do saldo de radiação, em $\mathrm{W} \mathrm{m}^{-2}$, está apresentada na Figura 7, com variação de valores próximos a $400 \mathrm{~W} \mathrm{~m}^{-2}$ (áreas claras) a superiores a $700 \mathrm{~W} \mathrm{~m}^{-2}$ (áreas escuras). Os maiores valores de $\mathrm{Rn}$ se apresentam nas áreas de cultivos irrigados onde existe vegetação densa e sem déficit de suprimento hídrico. $\mathrm{Na}$ Figura 8 está mostrada a distribuição espacial do fluxo de calor no solo, em W m$~^{-2}$, variando de valores inferiores a $70 \mathrm{~W} \mathrm{~m}^{-2}$ até superiores a $110 \mathrm{~W} \mathrm{~m}^{-2}$. Os menores valores de $\mathrm{G}$ são vistos nas áreas irrigadas, onde existe maior cobertura do solo e, por conseguinte, menor incidência de radiação solar à superfície. A distribuição espacial do fluxo de calor sensível, em $\mathrm{W} \mathrm{m}^{-2}$, está apresentada na Figura 9, a mesma tem valores variando entre $50 \mathrm{~W} \mathrm{~m}^{-2}$ e $350 \mathrm{~W} \mathrm{~m}^{-2}$. Os maiores valores de $\mathrm{H}$ se apresentam

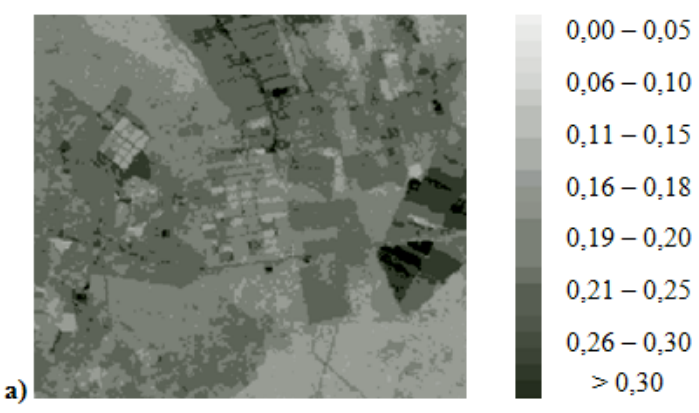

Figura 4 - Distribuição espacial do albedo da superfície.

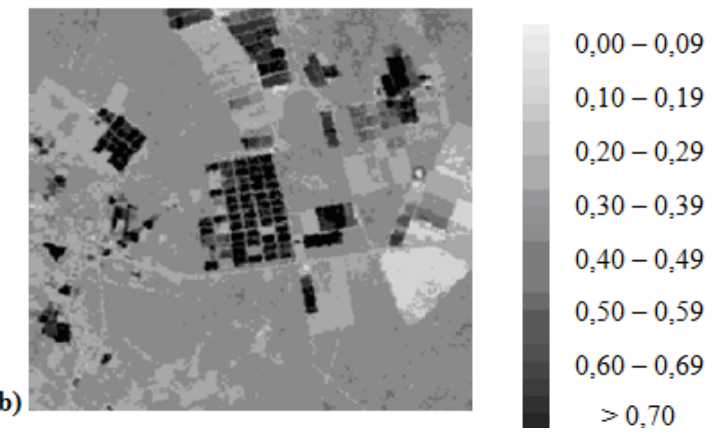

Figura 5 - Distribuição espacial do Índice de Vegetação por Diferença Normalizada (NDVI). 


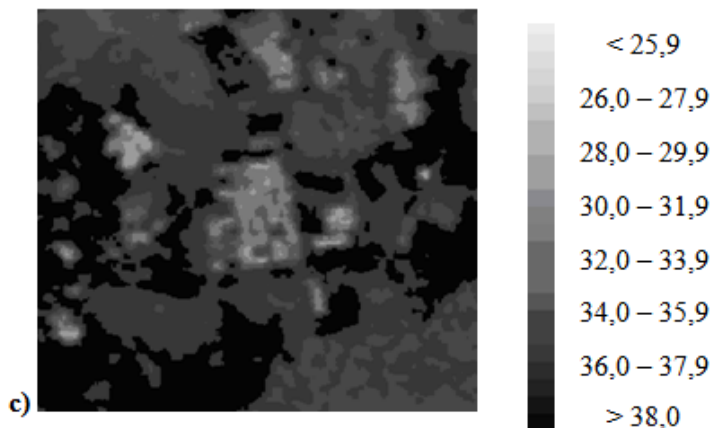

Figura 6 -Distribuição espacial da temperatura da superfície $\left({ }^{\circ} \mathrm{C}\right)$.

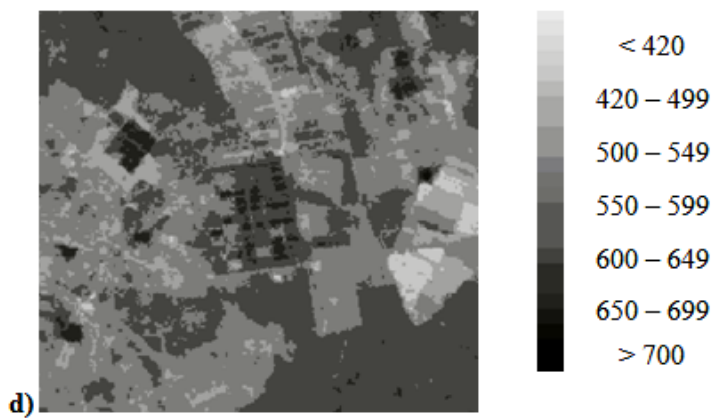

Figura 7 - Distribuição espacial do saldo de radiação $\left(\mathrm{W} \mathrm{m}^{-2}\right)$.

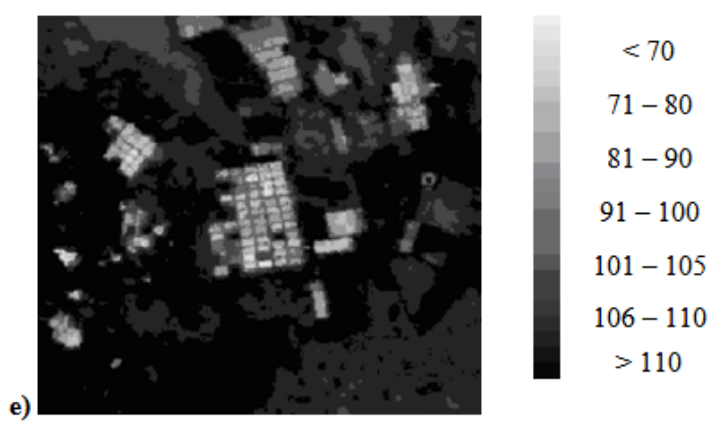

Figura 8 - Distribuição espacial do fluxo de calor no solo $\left(\mathrm{W} \mathrm{m}^{-2}\right)$.

nas áreas de solo exposto e de vegetação nativa, onde a maior parte da energia é utilizada para aquecer a superfície. Na Figura 10 , que apresenta valores variando de inferiores a $100 \mathrm{~W} \mathrm{~m}^{-2}$ até superiores a $400 \mathrm{~W} \mathrm{~m}^{-2}$, está mostrada a distribuição espacial do fluxo de calor latente, em $\mathrm{W} \mathrm{m}^{-2}$. Os maiores valores de LE se apresentam nas áreas de cultivos irrigados, onde a maior parte da energia disponível é utilizada no processo de evaporação do solo e transpiração das plantas, ou seja, na evapotranspiração.

\section{CONCLUSÕES}

O algoritmo S-SEBI apresentou-se como um bom estimador dos parâmetros: albedo, temperatura da superfície e dos fluxos de energia. Os resultados apresentaram concordância

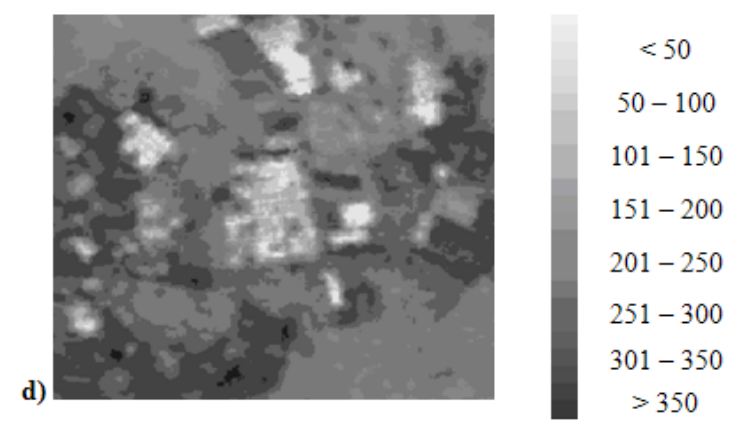

Figura 9-Distribuição espacial do fluxo de calor sensível $\left(\mathrm{W} \mathrm{m}^{-2}\right)$.

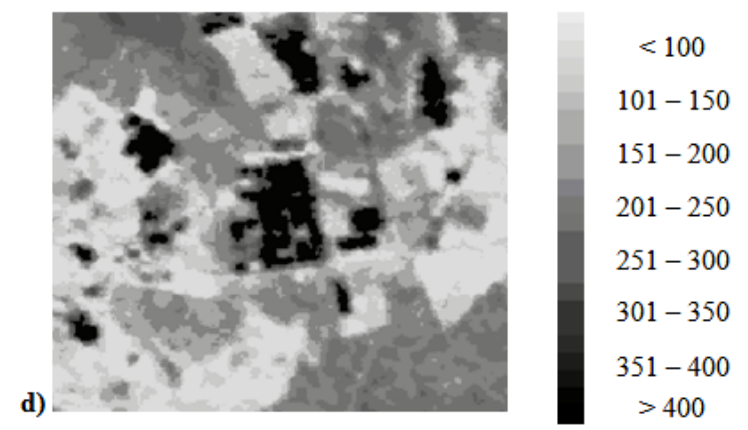

Figura 10 - Distribuição espacial do fluxo de calor latente $\left(\mathrm{W} \mathrm{m}^{-2}\right)$.

com estudos desenvolvidos em diferentes partes do mundo. A comparação dos resultados estimados pelo S-SEBI com os medidos na torre micrometeorológica mostrou que os parâmetros estimados pelo referido algoritmo estão em boa concordância com os medidos, evidenciando assim, que o S-SEBI aparece como uma ferramenta promissora na obtenção da distribuição espacial dos fluxos de energia à superfície em regiões semi-áridas. A principal desvantagem da metodologia proposta é o requerimento de valores extremos da superfície na imagem estudada e a necessidade de condições de céu-claro no momento da passagem do satélite. Outros métodos, como o SEBAL, mostram melhores resultados na obtenção dos fluxos de energia, entretanto, estes métodos necessitam da determinação do comprimento de rugosidade, que é uma tarefa difícil de ser obtida. Estudos futuros são necessários para confirmar os resultados apresentados, visto que nesse estudo as análises foram baseadas apenas em um caso, necessitando assim de um número maior de validações.

\section{AGRADECIMENTOS}

Os autores agradecem ao Conselho Nacional de Desenvolvimento Científico e Tecnológico $(\mathrm{CNPq})$ por financiar o projeto, fornecer bolsas de estudo e de pesquisa para os autores; à Fazenda Frutacor, que gentilmente cedeu suas áreas de cultivos para realização do experimento. Os autores também 
agradecem aos revisores anônimos que contribuíram com suas valiosas sugestões.

\section{REFERÊNCIAS BIBLIOGRÁFICAS}

ALLEN, R. G.; PEREIRA, L. S.; RAES, D.; SMITH, M. Crop evapotranspiration - Guidelines for computing crop water requirements - FAO Irrigation and drainage paper 56. Rome-Italy, 1998.

ALLEN, R.; TASUMI, M.; TREZZA, R. SEBAL (Surface Energy Balance Algorithms for Land) - Advanced Training and Users Manual - Idaho Implementation, version 1.0, 2002

ALLEN, R. G.; TASUMI, M.; MORSE, A.; TREZZA, R. A landsat-based energy balance and evapotranspiration model in Western US water rights regulation and planning. Irrigation and Drainage Systems, v. 19, 251-268 p., 2005.

BASTIAANSSEN, W. G. M. SEBAL-based sensible and latent heat fluxes in the irrigated Gediz Basin, Turkey. Journal of Hidrology, v. 229, p. 87-100, 2000.

BASTIAANSSEN, W. G. M.; MENENTI, M.; FEDDES, R. A.; HOLTSLAG, A. A. M. A remote sensing surface energy balance algorithm for land (SEBAL) 1. Formulation. Journal of Hydrology, v. 212-213, p.198-212. 1998.

BORONINA, A.; RAMILLIEN, G. Application of AVHRR imagery and GRACE measurements for calculation of actual evapotranspiration over the Quaternary aquifer (Lake Chad basin) and validation of groundwater models. Journal of Hydrology, v. 348, p. 98-109, 2008.

CHANDER, G.; MARKHAM, B. Revised Landsat-5 TM Radiometric Calibration Procedures ans Postcalibration Dynamic Ranges. IEEE Transactions on Geoscience and Remote Sensing. v. 41, p. 2674-2677, 2003.

FAN, L.; LIU, S.; BERNHOFER, C.; LIU, H.; BERGER, F. $\mathrm{H}$. Regional land surface energy fluxes by satellite remote sensing in the Upper Xilin River Watershed (Inner Mongolia, China). Theoretical and Applied Climatology, v. 88, p. 231-245, 2007.

GARCÍA, M.; VILLAGARCÍA, L.; CONTRERAS, S.; DOMINGO, F.; PUIGDEFÁBREGAS, J. Comparison of Three Operative Models for Estimating the Surface Water Deficit using ASTER Reflective and Thermal Data. Sensors, v. 7, p. 860-883, 2007.

GÓMEZ, M.; OLIOSO, A.; SOBRINO, J. A.; JACOB, F. Retrieval of evapotranspiration over the Alpilles/ReSeDA experimental site using airborne POLDER sensor and thermal camera. Remote Sensing of Environments, v. 96, p. 399-408, 2005.

HUETE, A. R. A soil adjusted vegetation index - SAVI. Remote Sensing of Environment, v. 25, p. 295-309, 1988.
NORMAN, J.; KUSTAS, W.; HUMES, K. A two-source approach for estimating soil and vegetation energy fluxes from observations of directional radiometric surface temperature. Agricultural and Forest Meteorology, v. 77, p. 263-293, 1995.

ROERINK, G. J.;SU,Z.; MENENTI, M.ASimple Remote Sensing Algorithm to Estimates the Surface Energy Balance. Physics and Chemistry of the Earth (B). n. 25, p. 147-157, 2000.

SANTOS, C.A. C.; SILVA, B. B. Estimativa da evapotranspiração da bananeira em região semi-árida através do algoritmo S-SEBI. Revista Brasileira de Agrometeorologia, v. 16, p. 9-20, 2008. SANTOS, C. A. C.; SILVA, B. B.; RAMANA RAO, T. V. Estimativa da ET diária utilizando os algoritmos SEBAL e S-SEBI. Revista Ciência e Natura, v. Especial, p. 71-74, 2007. SOBRINO, J. A.; GÓMEZ, M.; JIMÉNEZ-MUÑOZ, J. C.; OLIOSO, A. Application of a simple algorithm to estimate daily evapotranspiration from NOAA-AVHRR images for the Iberian Peninsula. Remote Sensing of Environment, v. 110, p. 139-148, 2007.

SOBRINO, J. A.; GÓMEZ, M.; JIMÉNEZ-MUÑOZ, J. C.; OLIOSO, A.; CHEHBOUNI, G. A simple algorithm to estimate evapotranspiration from DAIS data: Application to the DAISEX Campaigns. Journal of Hydrology, v. 315, p. 117-125, 2005.

SU, Z. The Surface Energy Balance System (SEBS) for estimation of turbulent heat fluxes. Hydrology and Earth System Sciences, v. 6, p. 85-99, 2002.

TANAKA, H; TAMAGAWA, I; ISHIKAWA, H; MA, Y.; HU, Z. Surface energy budget and closure of the eastern Tibetan Plateau during the GAME-Tibet IOP 1998. Journal of Hydrology, v. 283, p. 169-183, 2003.

TASUMI, M.; ALLEN, R. G. Satellite-Based ET mapping to assess variation in the ET with timing of crop development. Agricultural Water Management, v. 88, p. 54-62, 2007.

TASUMI, M.; TREZZA, R.; ALLEN, R. G.; WRIGHT, J. L. Operational aspects of satellite-based energy balance models for irrigated crops in the semi-arid U.S. Irrigation and Drainage Systems, v. 19, p. 355-376, 2005.

TEIXEIRA, A. H. C.; BASTIAANSSEN, W. G. M.; AHMAD, M. D.; BOS, M. G. Reviewing SEBAL input parameters for assessing evapotranspiration and water productivity for the Low-Middle São Francisco River basin, Brazil Part A: Calibration and validation. Agricultural and Forest Meteorology, v. 149, p. 462-476, 2009.

WEBB, E. K.; PEARMAN, G. I.; LEUNING, R. Correction of the flux measurements for density effects due to heat and water vapour transfer. Quarterly Journal of the Royal Meteorological Society, v. 106, p. 85-100, 1980.

WELIGEPOLAGE, K. Estimation of spatial and temporal distribution of evapotranspiration bay satellite remote 
sensing - A casa study in Hupselse Beek, The Netherlands. 2005, 114 p, Dissertation (Master of Science), International Institute for Geo-information Science and Earth Observation, Waganingen, Netherlands, 2005.
ZWART, S. J.; BASTIAANSSEN, W. G. M. SEBAL for detecting spatial variation of water productivity and scope for improvement in eight irrigated wheat systems. Agricultural Water Management, v. 89, 287-296 p., 2007. 